\title{
Experience of Health Complaints and Help Seeking Behavior in Employees Screened for Depressive Complaints and Risk of Future Sickness Absence
}

\author{
M. A. S. Lexis - N. W. H. Jansen - F. C. J. Stevens • \\ L. G. P. M. van Amelsvoort • IJ. Kant
}

Published online: 14 May 2010

(c) The Author(s) 2010. This article is published with open access at Springerlink.com

\begin{abstract}
Introduction The aim of this study was to examine the associations between on the one hand depressive complaints and risk of future sickness absence and on the other hand experience of health complaints and help seeking behavior in the working population. Methods Crosssectional data were used from employees working in the banking sector $(n=8,498)$. The screening instrument included measures to examine the risk of future sickness absence, depressive complaints and help seeking behavior. Results Of employees reporting health complaints, approximately $80 \%$ had already sought help for these complaints. Experience of health complaints and subsequent help seeking behavior differed between employees with mild to severe depressive complaints and employees at risk of future sickness absence. Experience of health complaints was highest in employees identified with both concepts (69\%) compared with employees identified at risk of future sickness absence only $(48 \%)$ and with mild to severe depressive complaints only (57\%). In those employees identified with one or both concepts and who had not sought help already, intention to seek help was about 50\%. Conclusions From a screening perspective, employees who do not experience health complaints or who do not have the intention to seek help may
\end{abstract}

M. A. S. Lexis $(\bowtie) \cdot$ N. W. H. Jansen

L. G. P. M. van Amelsvoort - IJ.Kant

CAPHRI School for Public Health and Primary Care,

Department of Epidemiology, Faculty of Health, Medicine

and Life Sciences, Maastricht University, P.O. Box 616,

6200 MD Maastricht, The Netherlands

e-mail: Monique.Lexis@epid.unimaas.nl

F. C. J. Stevens

Department of Educational Development and Research,

Faculty of Health, Medicine and Life Sciences,

Maastricht University, Maastricht, The Netherlands refuse participation in early intervention. This might be a bottleneck in the implementation of preventive interventions in the occupational health setting.

Keywords Health complaints · Help seeking · Preventive intervention · Employees

\section{Introduction}

In occupational health settings, interventions aimed at sickness absence of employees have increased during the past 20 years [1]. Most of the interventions focus on reintegration to work when sickness absence has already occurred [2-4]. Many studies have shown that treatment and reintegration have limited effects and that work resumption remains difficult [5-8]. Recently, a preventive strategy was developed in which employees at risk of future sickness absence receive early intervention to prevent future sickness absence [9]. Previous research by Kant et al. demonstrated that it is possible to identify employees at high risk of future sickness absence [10]. In an additional study it was demonstrated that preventive intervention among this group proved successful in reducing future sickness absence [9]. However, the latter study also revealed that the willingness and motivation of employees to participate in the preventive intervention varied substantially between subjects. Thus, having disposal of an adequate screening instrument as well as having a successful intervention does not necessarily result in successful prevention of sickness absence. An important reason for not participating in the preventive intervention could have been that employees, identified by the screening instrument at high risk of future sickness absence, did not experience or recognize any physical or mental health 
complaints at the time of the screening [9]. Consequently, these employees were not likely to display any help seeking behavior. This finding may imply a point of concern when conducting preventive interventions. Besides experience of health complaints, help seeking behavior may be influenced by many other factors as well, for example gender, age and lay referral [11-13]. According to the Health Belief Model (HBM) [14, 15], experience of health complaints is the most important determinant of help seeking behavior. Especially subjective experience of health complaints, defined as 'illness', determines help seeking behavior [16-20]. The HBM furthermore states that help seeking depends strongly on the individuals' perception that being ill may have serious consequences in terms of disease. Although the general principles of help seeking behavior apply to workers as well, the process may be different when compared with the general population. Work-related factors, such as work conditions and work environment, are mentioned as important factors that can contribute to development of health complaints and induce help seeking behavior. Interference of illness with work and disruption of the working situation may be an important reason to seek help in employees [20-22]. Employees might be expected to be aware of health complaints more promptly and seek help in a relatively early stage compared with the general population, since illness may lead to sickness absence and disability and become a threat to a person's income.

Recently, a randomized controlled trial (RCT) has been conducted to examine the efficacy of a preventive intervention in employees identified at high risk of sickness absence and with mild depressive complaints on the prevention of long-term sickness absence and major depression [Lexis et al., submitted]. The preventive intervention was specifically targeted at employees with depressive complaints, since depressive complaints were found to be highly prevalent in the working population and strongly associated with sickness absence [23]. Data of the RCT offered us the possibility to study the experience of health complaints and help seeking behavior among employees for two different concepts, that is, depressive complaints and the risk of future sickness absence. In case of screening for depressive complaints, the questionnaire assesses a person's experience of depressive symptoms. It may be expected that employees who experience depressive (=health) complaints at the time of completing the screening questionnaire will actually recognize these complaints and report them. In contrast with this, the concept of risk of sickness absence assesses a person's vulnerability for an event to happen in the future. This concept assesses a combination of factors that together determine the risk of future sickness absence. This multitude of factors does not necessarily have to be related to the experience of health complaints. It may occur that the increased risk can be mainly explained by work factors, such as having conflicts at work. In this situation it is likely that employees do not recognize health complaints at the moment of screening and do not recognize their 'being at risk' for a future event.

The aim of this study is to examine the experience of health complaints and help seeking behavior among the working population in relation to: (1) depressive complaints, (2) risk of sickness absence and (3) the combination of both concepts. It was hypothesized that employees identified with mild to severe depressive complaints will report to experience more health complaints compared with employees identified at high risk of future sickness absence. Moreover, it was hypothesize that in employees who do experience health complaints and who have not sought help already, those employees meeting the criteria for both concepts will have the highest intention to seek help. Additionally, specific characteristics of the employees were studied to investigate whether sub groups can be distinguished which might need a different guidance towards preventive intervention.

\section{Methods}

\section{Design and Study Population}

This cross-sectional study examined the relationship between on the one hand depressive complaints and risk of future sickness absence, and on the other hand experience of health complaints and help seeking behavior. The study was performed among employees working at a large banking company in the Netherlands and is part of a randomized controlled trial (RCT) that aims to investigate the efficacy of early intervention on the prevention of future sickness absence and major depression. For the current study, data from the screening questionnaires of the RCT were used. In 2007, 23,973 employees of the company received a screening questionnaire at their home address. The screening instrument included questionnaires to assess the risk of sickness absence, depressive complaints and included questions to examine the experience of health complaints and help seeking behavior. In the invitation letter, employees were asked to provide written informed consent. The informed consent covered the usage of the questionnaire data of the screening and the possibility to participate in the trial. After 2 weeks a reminder was sent to all non-respondents. Four separate batches were necessary to realize smooth processing of the large amount of returned questionnaires and to guarantee smooth enrolment of participants in the trial. Screening questionnaires were sent out in February $(n=7,000)$, March $(n=4,500)$, 
September $(n=6,196)$ and October $(n=6,277) 2007$. In total, 9,157 employees $(38.2 \%)$ responded to the screening instrument. 8,893 questionnaires were suitable for the analyses, 264 were discarded due to technical reasons, such as unreadable questionnaires or missing informed consent.

\section{Measurements}

The screening questionnaire contained questions to assess self-reported information on the exposure variables (1) risk of future sickness absence, (2) depressive complaints and to assess the outcomes variables (3) experience of health complaints and (4) help seeking behavior.

\section{Risk of Future Sickness Absence}

The Balansmeter (BM), which has been specifically developed for employees working in an office environment, was used to identify employees at high risk of future sickness absence. The BM was developed and validated in the Maastricht Cohort Study [10, 24] and includes 19 individual predictors for men and 22 for women. Due to overlapping predictors for men and women, the BM includes 34 multiple choice questions regarding demographics, work environment, private situation, (mental) health status, help seeking behavior, and sickness absence history. Using an algorithm based on the weighted factors of the individual items of the model, a total score can be calculated, with higher scores indicating a higher risk of future long-term sickness absence. In this study, in women a cut-off point with $87.9 \%$ specificity and $52.6 \%$ sensitivity was applied (score on BM -1.069) and in men specificity was $87.8 \%$ and sensitivity was $65.1 \%$ (score on BM $-1.944)$.

\section{Depressive Complaints}

The Hospital Anxiety and Depression (HAD) scale was used to assess the presence and severity of depressive complaints by self-report. The HAD is a 14 item self-report questionnaire, that was originally developed to indicate the presence and severity of both anxiety (HAD-A) and depression (HAD-D) separately $[25,26]$. Both the HAD-A and the HAD-D consist of 7 items and are scored on a four point Likert scale (0-3), resulting in a range of 0 tot 21 . In this study only the HAD-D was used. Although the HAD scale was originally developed to identify caseness of anxiety disorders and depression among patients in nonpsychiatric hospital clinics, the questionnaire was recently found to perform well in assessing the symptom severity and caseness of anxiety disorders and depression in somatic, psychiatric and primary care patients and in the general population [27] and also in the working population [28]. With respect to the cut-off values, we used the values suggested by Zigmond and Snaith [26]. A higher score on the HAD-D indicates more severe depressive complaints. Employees were defined as having mild to severe depressive complaints when they scored 8 points or higher on the HAD-D. Less than 8 points was defined as scoring in the normal range of the scale.

\section{Experience of Health Complaints and Help Seeking Behavior}

The screening instrument contained four questions on the outcomes experience of health complaints and help seeking behavior. The first question was: 'Do you currently experience any health complaints or health problems?' (yes/no). When the answer to this question was 'yes', the second question was: 'Have you already sought help for these complaints or problems?' (yes/no), and if the answer was yes, the third question was 'by whom?' (general practitioner and/or occupational physician and/or someone else). The fourth question investigated whether employees, who did not visit someone for their health complaints, have the intention to seek help in the near future (yes/no).

\section{Demographic, Health-Related and Work-Related Factors}

Employees provided information on gender, age, educational level, presence of a long-term illness (having the disease for more than 6 months), current sickness absence from work, experience of a life event during the past year, job insecurity and having an executive function through self-report in the questionnaire. Co-worker social support and skill competence were measured with a validated Dutch version of the self-administered Job Content Questionnaire (JCQ) [29, 30].

\section{In- and Exclusion Criteria of the Total Study Population}

Employees who responded to the screening questionnaire and who gave informed consent were included in the study. Those employees who were completely or partially on sick leave at the time of completing the screening questionnaire and female employees who were pregnant or on pregnancy leave were excluded from the analyses to avoid measuring help seeking behavior due to sickness absence and pregnancy-related health complaints.

\section{Definition of Three Study Populations}

Three populations were selected to study the concept of risk of sickness absence, the concept of depressive 
Fig. 1 Experience of health complaints and help seeking behavior

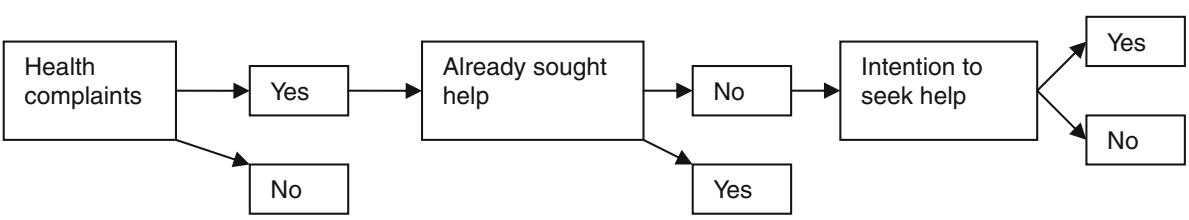

complaints and the concept of both risk of sickness absence and depressive complaints. For each population cases and non-cases were defined. The first study population was based on risk of future sickness absence (group A) and consisted of employees who scored above or below the predefined cut-off points (for women -1.069 , for men -1.944 ) of the BM, indicating 1,001 cases and 6,509 noncases. Scoring above the defined cut-off point indicates that the employee is at high risk of future sickness absence. The second study population was based on depressive complaints (group B). Scores above the cut-off point of 8 points on HAD-D indicated mild to severe depressive complaints (cases, $n=653$ ), scores below 8 indicated no to mild depressive complaints (non-cases, $n=7,224$ ). The third population was based on scores on both concepts (group C) and consisted of those employees who scored above (cases, $n=204$ ) both cut-off points of the BM and HAD-D and, in contrast, of those employees who scored below (non-cases, $n=7,202$ ) one or both cut-off points.

\section{Statistical Analysis}

First, independent samples $t$-tests and Chi-square tests were used to examine differences in experience of health complaints and intention to seek help in employees who responded to the screening instrument. These data were described for factors related to the experience of health complaints and help seeking behavior, such as demographic, health-related, private situation-related and workrelated factors.

Second, cross tabulations were calculated to describe differences in the experience of health complaints in employees identified (1) at risk versus not at risk of future sickness absence (group A), (2) with versus without mild to severe depressive complaints (group B) and (3) at risk of future sickness absence and with mild to severe depressive complaints versus employees not at risk of sickness absence and/or without mild to severe depressive complaints (group C).

Additionally, Chi-square tests were used to test differences in the experience of health complaints and help seeking behavior for several sub groups. Results were displayed for four potentially relevant sub groups, that is, severity of the complaints, gender, presence of long-term illness and educational level. Statistical analyses were performed using SPSS 15.0.

\section{Results}

Results for All Respondents on the Screening Questionnaire

Figure 1 displays the three questions on help seeking behavior included in the screening instrument. Table 1 presents the experience of health complaints and help seeking behavior among employees responding to the screening instrument. Approximately 2,311 (27\%) respondents reported to experience health complaints. Of these, $1,848(80 \%)$ employees already sought help for their health complaints. 1,293 employees sought help at a general practitioner, 341 at an occupational physician and 951 employees received help from someone else. Of the 463 employees who have not sought help already, $35 \%$ had the intention to seek help for the health complaints, whereas $46 \%$ had no intention. Regarding the descriptive characteristics of the study population, marked and statistically significant differences were found between the groups 'experience of health complaints no' (Table 1) and 'experience of health complaints yes and already sought help yes' with respect to demographics and factors regarding the private and work situation, except for skill discretion (=skill competence). Table 1 also presents the intention to seek help in employees who reported to experience health complaints but who had not sought help already. Most of the characteristics were comparable between those who reported to have and those who reported not to have the intention to seek help. However, in employees who had no intention to seek help, absence from work was significantly higher and more employees were having an executive position compared with those employees who did have the intention to seek help.

Association Between Risk of Sickness Absence, Depressive Complaints, Both Concepts and Experience of Health Complaints

Of all employees responding to the screening instrument, $13.3 \%$ was identified at high risk of future sickness absence and $8.3 \%$ was identified with mild to severe depressive complaints. Of the employees identified at high risk of sickness absence, $48 \%$ reported to experience health complaints, compared with $20 \%$ of the employees identified not at risk (group A) (Table 2). In contrast, the experience of 
Table 1 Experience of health complaints and help seeking behavior in respondents on the screening instrument

\begin{tabular}{|c|c|c|c|c|}
\hline \multirow[t]{4}{*}{$\begin{array}{l}\text { Health complaints NO } \\
(n=6,187)^{\mathrm{a}}\end{array}$} & \multirow{4}{*}{\multicolumn{2}{|c|}{$\begin{array}{l}\text { Health complaints } P \text {-value } \\
\text { YES }(n=2,311) \\
\text { Already sought } \\
\text { help YES } \\
(n=1,848)^{\mathrm{a}}\end{array}$}} & $\begin{array}{l}\text { Health complaints YES } \\
(n=2,311)\end{array}$ & $\begin{array}{l}\text { Health complaints Y } \\
(n=2,311)\end{array}$ \\
\hline & & & $\begin{array}{l}\text { Already sought } \\
\text { help NO }(n=463)\end{array}$ & $\begin{array}{l}\text { Already sought help } \\
\text { NO }(n=463)\end{array}$ \\
\hline & & & $\begin{array}{l}\text { Intention to seek } \\
\text { help YES }\end{array}$ & $\begin{array}{l}\text { Intention to seek } \\
\text { help NO }\end{array}$ \\
\hline & & & $(n=164)^{\mathrm{b}}$ & $(n=213)^{\mathrm{b}}$ \\
\hline
\end{tabular}

\begin{tabular}{|c|c|c|c|c|c|c|}
\hline \multicolumn{7}{|l|}{ Demographics } \\
\hline Male $(\%)$ & 52.1 & 44.9 & $<0.001$ & 50.0 & 49.8 & 0.964 \\
\hline Age, mean (SD) & $42.1(9.8)$ & $45.0(9.2)$ & $<0.001$ & $42.3(9.7)$ & $43.3(11.1)$ & 0.165 \\
\hline Educational level & & & $<0.001$ & & & 0.782 \\
\hline Low and medium $(\%)$ & 56.4 & 70.0 & & 61.9 & 63.4 & \\
\hline High $(\%)$ & 43.6 & 30.0 & & 38.1 & 36.6 & \\
\hline \multicolumn{7}{|l|}{ Health } \\
\hline Long-term illness (\% yes) & 15.0 & 62.6 & $<0.001$ & 36.2 & 37.7 & 0.760 \\
\hline Absent from work ( $\%$ yes) & 1.2 & 19.5 & $<0.001$ & 2.5 & 7.5 & 0.030 \\
\hline \multicolumn{7}{|l|}{ Private situation } \\
\hline $\begin{array}{l}\text { Experience of life-event } \\
\text { (\% yes) }\end{array}$ & 32.3 & 44.2 & $<0.001$ & 40.5 & 40.6 & 0.988 \\
\hline \multicolumn{7}{|l|}{ Work characteristics } \\
\hline $\begin{array}{l}\text { Coworker social support } \\
{[4-16]^{\mathrm{b}}, \text { mean }(\mathrm{SD})}\end{array}$ & $12.66(1.57)$ & $12.37(1.75)$ & $<0.001$ & $12.36(1.66)$ & $12.23(1.60)$ & 0.342 \\
\hline $\begin{array}{l}\text { Skill discretion }[12-48]^{\mathrm{b}} \text {, } \\
\quad \text { mean }(\mathrm{SD})\end{array}$ & $37.16(5.13)$ & $36.03(5.51)$ & 0.152 & $36.65(5.56)$ & $36.05(5.41)$ & 0.669 \\
\hline Job insecurity (\% yes) & 12.5 & 23.4 & $<0.001$ & 16.0 & 23.0 & 0.098 \\
\hline $\begin{array}{l}\text { Executive position } \\
\text { (\% yes) }\end{array}$ & 21.6 & 15.8 & $<0.001$ & 23.2 & 14.6 & 0.032 \\
\hline
\end{tabular}

${ }^{a}$ Total of cases does not equal 8,893 due to missing values

b Total of cases does not equal 463 due to missing values

Table 2 Association between risk of future sickness absence (SA) (group A), depressive complaints (group B), both risk of future SA and depressive complaints (group C) and experience of health complaints

\begin{tabular}{llll}
\hline & $\begin{array}{l}\text { Experience of health } \\
\text { complaints yes }\end{array}$ & $\begin{array}{l}\text { Experience of health } \\
\text { complaints no }\end{array}$ & Total \\
\hline At high risk of future SA & $484(48 \%)$ & $517(52 \%)$ & 1,001 \\
Not at risk of future SA & $1,293(20 \%)$ & $5,216(80 \%)$ & 6,509 \\
Total & 1,777 & 5,733 & 7,510 \\
Mild to severe depressive complaints & $370(57 \%)$ & $283(43 \%)$ & 653 \\
$\begin{array}{l}\text { No to mild depressive complaints } \\
\text { Total }\end{array}$ & $1,508(21 \%)$ & $5,716(79 \%)$ & 7,224 \\
At risk of future SA and mild to severe & 1,878 & 5,999 & 7,877 \\
$\quad$ depressive complaints & $140(69 \%)$ & $64(31 \%)$ & 204 \\
1-At risk of future SA and/or mild to severe & $1,609(22 \%)$ & $5,593(78 \%)$ & $<0.001$ \\
$\quad$ depressive complaints & 1,749 & 5,657 & 7,202 \\
Total & & 7,406 \\
\hline
\end{tabular}

Totals of cases do not equal 8,893 due to missing values 
health complaints in employees identified with depressive complaints was higher (57\%), in comparison with $21 \%$ of the employees without depressive complaints (group B). When looking at employees meeting the criteria for both concepts (group C), 69\% reported to experience health complaints.

We additionally studied the effect of cut-off points based on a higher specificity for both risk of sickness absence (94.4\% for men and $94.7 \%$ for women) and depressive complaints (HAD-D $\geq 11$ ) on the experience of health complaints, since the choice of a cut-off point is relatively arbitrary and therefore may have influenced the results (specific data not shown). When applying these cutoff points, experience of health complaints for risk of future sickness absence increased to $58 \%$, for depressive complaints to $68 \%$ and for both concepts together to $75 \%$.

Characteristics of the Three Study Populations

Table 3 presents help seeking behavior in employees selected by the screening instrument as eligible candidates for participation in preventive intervention. Eligible candidates were employees identified at risk of sickness absence (group A), with mild to severe depressive complaints (group B) or meeting the criteria of both concepts simultaneously (group C). In employees meeting the criteria of both concepts (group C), the experience of health complaints (140/204) and the intention to seek help in employees who have not sought help already for these health complaints (16/30) was found to be highest compared with groups $\mathrm{A}$ and $\mathrm{B}$.
Statistically significant differences were found in the experience of health complaints when looking at the severity of complaints and presence of a long-term illness in all three concepts. Significant gender differences were found only for risk of future sickness absence, with more men than women experiencing health complaints. Significant differences in educational level were found only for depressive complaints, with employees having a low or medium educational level experiencing more health complaints. When looking at 'already sought help', among employees at risk of future sickness absence and among employees with mild to severe depressive complaints, those employees having a long-term illness have sought significantly more help for their health complaints already compared with those without a long-term illness. Of the employees who reported to experience health complaints and who have not sought help already, the intention to seek help proved rather low, approximately $50 \%$ in all three concepts. No statistically significant differences were found for the intention to seek help with regard to the severity of complaints, gender, presence of a long-term illness and educational level.

\section{Discussion}

\section{Main Findings}

To our knowledge, this the first study that aimed at quantifying help seeking behavior specifically in the working

Table 3 Help seeking behavior in employees identified at risk of future sickness absence (SA), with mild to severe depressive complaints and both at risk of future SA and with mild to severe depressive complaints

\begin{tabular}{|c|c|c|c|c|c|c|c|c|c|}
\hline & \multicolumn{3}{|c|}{ Experience of health complaints } & \multicolumn{3}{|c|}{ Already sought help } & \multicolumn{3}{|c|}{ Intention to seek help } \\
\hline & No & Yes & $P$-value & Yes & No & $P$-value & Yes & No & $P$-value \\
\hline Risk of SA $(\mathrm{BM} \geq 13,3)$ & 517 & 484 & $<0.001$ & 404 & 80 & $<0.001$ & 31 & 37 & 0.875 \\
\hline Severity $(\mathrm{BM} \geq 6,6)(\%$ yes $)$ & 42.0 & 62.6 & $<0.001$ & 63.1 & 60.0 & 0.598 & 61.3 & 56.8 & 0.705 \\
\hline Male (\% yes) & 73.9 & 66.1 & 0.007 & 67.1 & 61.3 & 0.314 & 58.1 & 62.2 & 0.731 \\
\hline Long-term illness (\% yes) & 29.0 & 66.8 & $<0.001$ & 70.0 & 51.3 & 0.001 & 38.7 & 55.6 & 0.169 \\
\hline Educational level high (\% yes) & 19.0 & 19.0 & 0.986 & 18.2 & 23.1 & 0.313 & 26.7 & 19.4 & 0.486 \\
\hline Depressive complaints (HAD-D $\geq 8$ ) & 283 & 370 & $<0.001$ & 259 & 111 & $<0.001$ & 41 & 53 & 0.782 \\
\hline Severity (HAD $\geq 11)$ (\% yes) & 21.2 & 34.3 & $<0.001$ & 35.9 & 30.6 & 0.327 & 29.3 & 28.3 & 0.918 \\
\hline Male (\% yes) & 51.2 & 53.0 & 0.660 & 50.6 & 58.6 & 0.159 & 53.7 & 58.5 & 0.639 \\
\hline Long-term illness (\% yes) & 19.4 & 54.9 & $<0.001$ & 64.7 & 44.3 & $<0.001$ & 46.3 & 38.0 & 0.422 \\
\hline Educational level high (\% yes) & 35.4 & 26.9 & 0.024 & 25.7 & 29.5 & 0.461 & 40.0 & 24.5 & 0.117 \\
\hline $\begin{array}{l}\text { At risk of SA \& depressive complaints } \\
\quad(\mathrm{BM} \geq 13,3, \mathrm{HAD}-\mathrm{D} \geq 8)\end{array}$ & 64 & 140 & $<0.001$ & 105 & 35 & 0.484 & 16 & 14 & 0.321 \\
\hline $\begin{array}{l}\text { Severity }(\mathrm{BM} \geq 6,6 \& \text { HAD-D } \geq 8) \\
\quad(\% \text { yes })\end{array}$ & 53.1 & 72.9 & 0.006 & 75.2 & 65.7 & 0.273 & 62.5 & 64.3 & 0.919 \\
\hline Male (\% yes) & 57.8 & 63.6 & 0.432 & 64.8 & 60.0 & 0.612 & 50.0 & 57.1 & 0.696 \\
\hline Long-term illness ( $\%$ yes) & 25.4 & 63.8 & $<0.001$ & 67.0 & 54.5 & 0.198 & 56.3 & 46.2 & 0.588 \\
\hline Educational level high (\% yes) & 21.0 & 16.2 & 0.414 & 16.5 & 15.2 & 0.856 & 26.7 & 7.7 & 0.191 \\
\hline
\end{tabular}


population, by studying the associations between employees identified with depressive complaints and/or at risk of future sickness absence and their experience of health complaints and subsequent help seeking behavior.

From the general population it is known that only a relatively small part of the population experiencing symptoms/health complaints seeks help, called the clinical iceberg [31]. In our study, approximately $80 \%$ of the employees who reported to experience health complaints had already sought help for their health complaints. This high percentage may be explained by the theory of illness behavior and the HBM, both stating that help seeking behavior is strongly affected by the possible negative consequences of health complaints. For employees, health complaints may have serious consequences for functioning at work or may even result in losing one's job, where loss of income then may become a serious threat. This may induce help seeking behavior in this population. Of the employees who already sought help, $18.5 \%$ received help from an occupational physician of the banking company. This may have been influenced by the nature of the occupational health services (OHS) of the banking company under study. The OHS have regular contacts with their employees during the year and can be characterized as being easily accessible for the employees.

Regarding the results of all respondents on the screening instrument, significant differences were found in the characteristics of employees who did and who did not experience health complaints. Experience of health complaints was significantly higher in female employees, in employees having a higher age, a lower educational level, a long-term illness, which is in line with the literature about illness behavior [12, 32-34]. Experience of health complaints and the intention to seek help was higher in employees having an executive position. Experience of health complaints was also higher in those employees who reported high job insecurity. This may be explained by the fact that health complaints may have more (serious) adverse consequences in case of having an executive function or having fear to lose one's job.

Of all employees responding to the screening instrument, approximately $30 \%$ indicated to experience health complaints. Regarding the results of the employees screened for risk of future sickness absence (group A), for depressive complaints (group B), and for both concepts (group C), it was expected that many employees screened 'positively' would actually report to experience health complaints. However, the results demonstrated that this did not turn out to be the case. When comparing the results of the three groups with the results of all respondents together, experience of health complaints increased among the employees identified at risk of future sickness absence $(48 \%)$ and also among those identified with mild to severe depressive complaints (57\%). Experience of health complaints was highest among those identified with both concepts $(69 \%)$. Especially for group B and group C, more employees were expected to have reported health complaints. However, from those employees identified with mild to severe depressive complaints, still $43 \%$ of the respondents did not report to experience health complaints at that moment. This might be due to the relatively mild level of health complaints the screening instrument aimed at.

Employees identified with more severe complaints and employees with a long-term illness reported to experience significantly more health complaints in each of the three concepts. Employees with a long-term illness had also sought significantly more help already. Significantly more health complaints were experienced by those employees identified with depressive complaints with a low or medium educational level. These findings are in line with the literature, as mentioned earlier. However, in employees identified at risk of sickness absence men were found to experience more health complaints than women. This is in contrast with studies conducted in the general population and also in the working population, in which women usually tend to report more health complaints [33-36]. As compared with the general population, differences occurred regarding experience of health complaints and help seeking behavior in the working population. First, people being in poor health are not likely to enter the labor force. Second, employees who develop (serious) bad health over time are likely to leave the labor force at a certain point in time. For example, women who have a long-term illness may not even start working or they may quit working when they get ill. This is different for men, who more often occupy the role of breadwinner and therefore need to start working and/or remain working. An earlier study observed that the vast majority of men with a small part-time job reported to have a long-term illness [37]. These selection effects may also explain differences in prevalence of especially the more severe health complaints in the general and working population, since employees with more severe complaints may not enter at all or may have already left the labor force. This may result in an underestimation of severe health complaints in the working population. Consequently, a higher prevalence of people with severe health complaints is more likely to be observed in the general population.

Approximately $70 \%$ of all employees identified with both mild to severe depressive complaints and at risk of sickness absence indicated to recognize health complaints. This implies that still $30 \%$ of the employees screened positively on both concepts indicated not to experience health complaints. Regarding the intention to seek help, in all three concepts the intention to seek help was about $50 \%$. Of all employees who reported to experience health 
complaints and who have not already sought help for these complaints, $50 \%$ indicated to have no intention to seek help. These two findings are of special importance from the perspective of early intervention. The main assumption of this study was that experience of health complaints is a prerequisite for help seeking behavior. Additionally, experience of health complaints did not automatically imply an intention to seek help. When these employees are offered the possibility to participate in early intervention, no experience of health complaints or not having the intention to seek help may constitute a bottleneck in participation in early intervention.

\section{Methodological Considerations}

The following issues should be kept in mind when interpreting the results of this study.

We studied the association between mild to severe depressive complaints, risk of future sickness absence and the experience of health complaints. The screening questionnaire investigated help seeking behavior due to health complaints. However, we cannot state with certainty that the reported health complaints were caused by depressive complaints or by the factors included to measure risk of sickness absence, since data on the reasons for the health complaints was not available. These reasons may include factors such as conflicts at work, marital problems or financial problems. Because the design of the study is cross-sectional, conclusions about causes and effects can not be drawn from these results.

Data on the intention to seek help for health complaints were assessed by the screening questionnaire. However, data on the actual participation of employees allocated to the intervention were not available. So, the relationship between intention to seek help and actual participation in preventive intervention could not be studied.

The cut-off point of the HAD-D was set at 8 points or higher indicating a range from mild to severe depressive complaints. Because this range is very broad, differences in the experience of health complaints within the group of depressive complaints may certainly appear. This may also apply for risk of sickness absence, since a relatively mild cut-off point was chosen. Since the choice for a cut-off point always remains arbitrary, we explored the impact of cut-off points based on a higher specificity for both concepts. Experience of health complaints increased indeed, but reached in neither case over $75 \%$. When screening for both being at risk of sickness absence and depressive complaints there is still $25 \%$ of the respondents who do not report health complaints.

When applying a screening instrument, misclassification of participants is likely to occur, resulting in false-positive or false-negative classification. In case of risk of sickness absence, a high specificity was preferred when the questionnaire was developed. For men specificity was $94.4 \%$ and in women $94.7 \%$. Thus, approximately $5.5 \%$ of all participants will be classified as false-positives, employees who are not at risk actually, but nevertheless classified at risk by the screening instrument. From research on the development of the HAD-scale by Zigmond and Snaith, it can be derived that the HAD-D has a very high specificity as well [26]. Our results showed that $52 \%$ of the employees at risk of sickness absence and $43 \%$ of the employees with mild to severe depressive complaints do not experience health complaints. This indicates that these percentages could only be explained for a small part by misclassification by the screening instrument.

Although many important factors often mentioned in the literature to be associated with illness behavior were included in the analyses, some of them were or could not be included in our questionnaire, such as data on ethnicity and cultural background.

The majority of the employees screened 'positively' for depressive complaints, risk of future sickness absence and meeting the criteria for both concepts, did already seek help for their health complaints. These employees have already undertaken action themselves to solve their problems and therefore they do not belong to the target group the preventive strategy aims at.

\section{Implications for Future Research}

The present study gained insight in the background of help seeking behavior of employees identified as eligible candidates for participation in preventive intervention. The results found may contribute to the explanation of the relatively low participation of employees in preventive intervention. Our results demonstrated that most of the employees reporting health complaints already sought help for these complaints. Of the employees identified at risk of future sickness absence, with mild to severe depressive complaints or identified with both concepts, still 30-50\% did report not to experience health complaints and of these approximately $50 \%$ did not have an intention to seek help. This group of employees may include those most likely to refuse participation in preventive intervention, while intervention could be beneficial in reducing the risk of future sickness absence and/or depressive complaints. Professionals working in occupational health care should take this into account in case of early intervention.

Besides the experience of health complaints, there are many other factors that may determine participation and successful implementation of preventive interventions, such as the ability to offer voluntary participation, the possibility of participation in and after working hours, a location within easy reach and for example the person's belief in the 
effectiveness of the intervention. Participation may also depend on many other factors as described in the Health Belief Model, such as personality, self-efficacy, cues to action and an individual's personal considerations, for example fear to lose one's job. Employees may refuse participation because they do not want their problems become noticeable for the boss and/or colleagues. Since our study was carried out within one banking company, the influence of factors such as the corporate culture could not be investigated, but might have an important role as well. All these factors may contribute remarkably to the willingness to participate in preventive intervention; however, they could not all be included in the current study. To further improve implementation of preventive interventions, the aim for future research could be on studying factors that determine help seeking behavior, to get more insight in the concept of help seeking behavior among employees. Another possibility could be to focus on those employees who do experience health complaints and to study which factors have an influence on actual participation, in order to optimize participation in preventive intervention.

Acknowledgments This study was financially supported by the Netherlands Organisation for Health Research and Development (ZonMw), grant no. 62200024, by CAPHRI School for Public Health and Primary Care, Maastricht, the Netherlands and by the Occupational Health Services 'Beter' (ABN AMRO Arbo Services), Amsterdam, the Netherlands.

Open Access This article is distributed under the terms of the Creative Commons Attribution Noncommercial License which permits any noncommercial use, distribution, and reproduction in any medium, provided the original author(s) and source are credited.

\section{References}

1. Noordik E, van Dijk FJ, Nieuwenhuijsen K, van der Klink JJ. Effectiveness and cost-effectiveness of an exposure-based returnto-work programme for patients on sick leave due to common mental disorders: design of a cluster-randomized controlled trial. BMC Public Health. 2009;9:140.

2. Bultmann U, Sherson D, Olsen J, Hansen CL, Lund T, Kilsgaard J. Coordinated and tailored work rehabilitation: a randomized controlled trial with economic evaluation undertaken with workers on sick leave due to musculoskeletal disorders. J Occup Rehabil. 2009;19:81-93.

3. van der Klink JJ, Blonk RW, Schene AH, van Dijk FJ. Reducing long term sickness absence by an activating intervention in adjustment disorders: a cluster randomised controlled design. Occup Environ Med. 2003;60:429-37.

4. van Oostrom SH, Anema JR, Terluin B, Venema A, de Vet HC, van Mechelen W. Development of a workplace intervention for sick-listed employees with stress-related mental disorders: intervention mapping as a useful tool. BMC Health Serv Res. 2007;7:127.

5. Brouwers EP, Tiemens BG, Terluin B, Verhaak PF. Effectiveness of an intervention to reduce sickness absence in patients with emotional distress or minor mental disorders: a randomized controlled effectiveness trial. Gen Hosp Psychiatry. 2006;28: 223-9.

6. Huibers MJ, Beurskens AJ, Van Schayck CP, Bazelmans E, Metsemakers JF, Knottnerus JA, et al. Efficacy of cognitivebehavioural therapy by general practitioners for unexplained fatigue among employees: randomised controlled trial. $\mathrm{Br} \mathrm{J}$ Psychiatry. 2004;184:240-6.

7. Janssen N, van den Heuvel WP, Beurskens AJ, Nijhuis FJ, Schroer CA, van Eijk JT. The demand-control-support model as a predictor of return to work. Int J Rehab Res. 2003;26:1-9.

8. Lander F, Friche C, Tornemand H, Andersen JH, Kirkeskov L. Can we enhance the ability to return to work among workers with stress-related disorders? BMC Public Health. 2009;9:372.

9. Kant I, Jansen NW, van Amelsvoort LG, van Leusden R, Berkouwer A. Structured early consultation with the occupational physician reduces sickness absence among office workers at high risk for long-term sickness absence: a randomized controlled trial. J Occup Rehabil. 2008;18:79-86.

10. Kant IJ, Jansen NW, van Amelsvoort LG, Swaen GM, van Leusden R, Berkouwer A. Screening questionnaire Balansmeter proved successful in predicting future long-term sickness absence in office workers. J Clin Epidemiol. 2009;62:408-14.

11. Judd F, Komiti A, Jackson H. How does being female assist helpseeking for mental health problems? Aust N Z J Psychiatry. 2008; 42:24-9.

12. Mackenzie CS, Scott T, Mather A, Sareen J. Older adults' helpseeking attitudes and treatment beliefs concerning mental health problems. Am J Geriatr Psychiatry. 2008;6:1010-9.

13. Rudell K, Bhui K, Priebe S. Do 'alternative' help-seeking strategies affect primary care service use? A survey of help-seeking for mental distress. BMC Public Health. 2008;8:207.

14. Becker MH. Health belief model and personal health behavior. Health Educ Monogr. 1974;2:324-473.

15. Rosenstock IM. Why people use health services. Milbank Mem Fund Q. 1966;44:94-127.

16. Kasl SV, Cobb S. Health behavior, illness behavior, and sick role behavior. Arch Environ Health. 1966;12:246-66.

17. Mechanic D. The concept of illness behavior. J Chronic Dis. 1962;15:189-94.

18. Shaw C. A framework for the study of coping, illness behaviour and outcomes. J Adv Nurs. 1999;29:1246-55.

19. Shaw C, Brittain K, Tansey R, Williams K. How people decide to seek health care: a qualitative study. Int J Nurs Stud. 2008;45: 1516-24.

20. Wikman A, Marklund S, Alexanderson K. Illness, disease, and sickness absence: an empirical test of differences between concepts of ill health. J Epidemiol Community Health. 2005;59: $450-4$.

21. Mechanic D. Sociological dimensions of illness behavior. Soc Sci Med. 1995;41:1207-16.

22. Zola IK. Pathways to the doctor: from person to patient. Soc Sci Med. 1973;7:677-89.

23. Lexis MA, Jansen NW, van Amelsvoort LG, van den Brandt PA, Kant I. Depressive complaints as a predictor of sickness absence among the working population. J Occup Environ Med. 2009;51: 887-95.

24. Kant IJ, Bultmann U, Schroer KA, Beurskens AJ, Van Amelsvoort LG, Swaen GM. An epidemiological approach to study fatigue in the working population: the Maastricht Cohort Study. Occup Environ Med. 2003;60:32-9.

25. Snaith RP. The hospital anxiety and depression scale. Health Qual Life Outcomes. 2003;1:29.

26. Zigmond AS, Snaith RP. The hospital anxiety and depression scale. Acta Psychiatr Scand. 1983;67:361-70.

27. Spinhoven P, Ormel J, Sloekers PP, Kempen GI, Speckens AE, Van Hemert AM. A validation study of the Hospital Anxiety and 
Depression Scale (HADS) in different groups of Dutch subjects. Psychol Med. 1997;27:363-70.

28. Andrea H, Bultmann U, Beurskens AJ, Swaen GM, van Schayck $\mathrm{CP}$, Kant IJ. Anxiety and depression in the working population using the HAD Scale-psychometrics, prevalence and relationships with psychosocial work characteristics. Soc Psychiatry Psychiatr Epidemiol. 2004;39:637-46.

29. Karasek R, Brisson C, Kawakami N, Houtman I, Bongers P, Amick B. The Job Content Questionnaire (JCQ): an instrument for internationally comparative assessments of psychosocial job characteristics. J Occup Health Psychol. 1998;3:322-55.

30. Karasek RA. The Job Content Questionnaire and User's Guide (version 1.1). Los Angeles, CA: Department of Industrial and Systems Engineering, University of Southern California; 1985.

31. Hannay DR. The 'iceberg' of illness and 'trivial' consultations. J R Coll Gen Pract. 1980;30:551-4.

32. Mechanic D. Health and illness behavior and patient-practitioner relationships. Soc Sci Med. 1992;34:1345-50.
33. Moller-Leimkuhler AM. Barriers to help-seeking by men: a review of sociocultural and clinical literature with particular reference to depression. J Affect Disord. 2002;71:1-9.

34. Raviv A, Sills R, Raviv A, Wilansky P. Adolescents' helpseeking behaviour: the difference between self- and other-referral. J Adolesc. 2000;23:721-40.

35. Hensing G, Alexanderson K, Allebeck P, Bjurulf P. Sick-leave due to psychiatric disorder: higher incidence among women and longer duration for men. Br J Psychiatry. 1996;169:740-6.

36. Mojtabai R. Social comparison of distress and mental health helpseeking in the US general population. Soc Sci Med. 2008;67: 1944-50.

37. Jansen N, Kant I, van Amelsvoort L, Nijhuis F, van den Brandt P. Need for recovery from work: evaluating short-term effects of working hours, patterns and schedules. Ergonomics. 2003;46: 664-80. 